\title{
The Relationship between Neuropsychiatric Symptoms and Default-Mode Network Connectivity in Alzheimer's Disease
}

\author{
Jung Suk Lee ${ }^{1,2} \bowtie$, Jong Hun Kim ${ }^{1,3}$, and Seon-Koo Lee ${ }^{4}$ \\ ${ }^{1}$ Dementia Center, National Health Insurance Service Ilsan Hospital, Goyang, Republic of Korea \\ ${ }^{2}$ Department of Psychiatry, National Health Insurance Service Ilsan Hospital, Goyang, Republic of Korea \\ ${ }^{3}$ Department of Neurology, National Health Insurance Service Ilsan Hospital, Goyang, Republic of Korea \\ ${ }^{4}$ Department of Psychiatry, Bundang Jesaeng Hospital, Seongnam, Republic of Korea
}

Objective Neuropsychiatric symptoms of dementia are prevalent and extremely burdening for the patient and caregivers, but the underlying mechanism of these symptoms has not been investigated. This study aimed to investigate the relationship between neuropsychiatric symptoms and default-mode functional connectivity in Alzheimer's disease.

Methods Neuropsychiatric symptoms were assessed using the Neuropsychiatric Inventory. Functional magnetic resonance imaging was conducted on seventy patients with Alzheimer's disease during rest. We conducted a seed-based functional connectivity analysis to identify anterior and posterior default-mode networks (DMN). Seeds were the medial prefrontal cortex (Montreal Neurological Institute 12, 51, 36; seed radius $=3 \mathrm{~mm}$ ) for the anterior DMN and the precuneus (Montreal Neurological Institute $-6,-63,27$; seed radius $=3 \mathrm{~mm}$ ) for the posterior DMN We then correlated the scores on neuropsychiatric inventory syndromes (apathy, hyperactivity, affective, and psychosis syndrome) with maps of connectivity in the default-mode network.

Results There was a significant correlation between decreased connectivity in the medial prefrontal cortex of the anterior defaultmode network and hyperactivity (agitation, irritability, aberrant motor behavior, euphoria, and disinhibition) syndrome ( $\mathrm{p}<0.05$, family wise error cluster-level corrected).

Conclusion Our study demonstrated that hyperactivity syndrome is related to hypoconnected default-mode network in Alzheimer's disease. This finding suggests that specific network alterations are associated with certain neuropsychiatric syndromes.

Psychiatry Investig 2020;17(7):662-666

Key Words Neuropsychiatric symptoms, Hyperactivity, Alzheimer's disease, Default-mode network, Medial prefrontal cortex, Functional magnetic resonance imaging.

\section{INTRODUCTION}

Neuropsychiatric symptoms (NPS), such as agitation, aggression, delusions, hallucinations, depression, anxiety, apathy, disinhibition, and sleep disturbances, are among the earliest sings of neurocognitive disorders. ${ }^{1}$ NPS are highly prevalent in patients with Alzheimer's disease and one of NPS are reported in 60 to 90 percent of patients with dementia. ${ }^{2-4}$ NPS can be of great burden not only for patients with

\footnotetext{
Received: January 9, 2020 Revised: April 1, 2020

Accepted: April 7, 2020

$\triangle$ Correspondence: Jung Suk Lee, MD, PhD

Department of Psychiatry, National Health Insurance Service Ilsan Hospital, 100 Ilsan-ro, Goyang 10444, Republic of Korea

Tel: +82-31-900-0269, Fax: +82-31-900-0343, E-mail: thanato9@nhimc.or.kr

(c) This is an Open Access article distributed under the terms of the Creative Commons Attribution Non-Commercial License (https://creativecommons.org/licenses/bync/4.0) which permits unrestricted non-commercial use, distribution, and reproduction in any medium, provided the original work is properly cited.
}

dementia but also for caregivers. ${ }^{5,6}$ Further, NPS can be associated with faster progression from early dementia to severe dementia and death. ${ }^{7}$

So far there have been a lot of studies on the biological mechanism of NPS, but the neurobiology underlying these symptoms are poorly understood. Previous neuroimaging studies using magnetic resonance imaging (MRI), positron emission tomography (PET), and single-photon emission computed tomography (SPECT) have shown that apathy in patients with Alzheimer's disease were related to the orbitofrontal cortex, the anterior cingulate cortex and the inferior temporal cortex..$^{8-11}$ In addition, studies have shown that structural abnormalities in white matter such as increase fractional anisotropy were associated with NPS in Alzheimer's disease. ${ }^{12,13}$ However, much research has not been done on the relationship between NPS and functional connectivity in the brain.

Recent developments in functional MRI have revealed a 
disorder of functional connectivity of the brain in dementia. Functional connectivity means that spatially separated brain regions have a close functional correlation. This functional connectivity is observed when performing any cognitive task but also during rest-state or sleep. The abnormalities in functional connectivity eventually lead to functional abnormalities in each brain region, which can result in cognitive dysfunction and NPS. A recent study examined the relationship between resting functional connectivity and NPS in patients with Alzheimer's disease, and it has shown that functional connectivity between the anterior cingulate cortex and the insula was associated with hyperactivity syndrome including agitation and disinhibition. ${ }^{14}$

Among the brain networks, the default mode network is known to be particularly involved and affected in Alzheimer's disease. ${ }^{15}$ The DMN is preferentially activated during internal tasks such as self-reflection, envisioning the future, and remembering the past, while it is deactivated during external goal-oriented tasks such as visual attention and working memory tasks. ${ }^{16}$ The DMN could further been divided into 2 major functional subnetworks: the anterior DMN, which centers on the medial prefrontal cortex, and the posterior DMN including the precuneus, the posterior cingulate cortex, and the lateral parietal cortex. ${ }^{17}$ These subnetworks have been demonstrated to be differentially affected during the progression of Alzheimer's disease, with the posterior DMN showing reduced connectivity in the early stages, whilst the anterior DMN show increased connectivity which diminishes with disease progression. ${ }^{18}$ However, there are currently no data on how the DMN and NPS are related in patients with Alzheimer's disease.

The purpose of this study is to elucidate the relationship between NPS and resting functional connectivity in patients with Alzheimer's disease. We predicted that alterations in the DMN would be related to subsyndromes of NPS in patients with Alzheimer's disease.

\section{METHODS}

\section{Subjects}

Seventy patients with Alzheimer's disease participated in the study. The subjects were recruited from outpatient clinic and diagnosed with probable Alzheimer's disease according to the National Institute of Neurological and Communicative Disorders and Stroke and Alzheimer's Disease and Related Disorders Association. ${ }^{19}$ Interviews for diagnosis and evaluation were conducted by neurologists or neuropsychologists. Exclusion criteria were 1) patients with degenerative brain diseases such as Lewy body dementia, Parkinson's disease, Huntington's chorea, Down's syndrome, Creutzfeldt-
Jakob disease, 2) history of head trauma with loss of consciousness or mental retardation, 3) previous history of cerebrovascular disease or stroke on brain magnetic resonance imaging, and 4) current or past psychiatric illness. This study has been approved by the Institutional Review Board, and all participants have given written consent after fully understanding the study (2016-03-041).

\section{Measures}

Global cognitive status was assessed by Korean Mini-Mental State Examination (K-MMSE) ${ }^{20}$ and clinical dementia rating $(\mathrm{CDR}) .^{21}$ In order to evaluate NPS, the Korean version of the Neuropsychiatric Inventory (NPI) ${ }^{22}$ was used to interview caregivers of patients with dementia. The NPI consists of the following 12 neuropsychiatric domains: hallucinations, delusions, agitation/aggression, depression, anxiety, irritability, disinhibition, euphoria, apathy, aberrant motor behavior, change in night-time sleep behavior, and changes in appetite and eating. The NPI assesses the frequency and severity of 12 behavioral symptoms, and at the same time, the degree of distress of the caregiver. The scores were calculated by multiplying the frequency of the symptoms (from 1 to 4 ) by the intensity (from 1 to 3 ). The total score ranges from 0 to 144, with higher scores indicating more severe NPS. According to a previous study, ${ }^{23}$ NPS were divided into four subsyndromes: apathy, hyperactivity (the sum of agitation, disinhibition, irritability, euphoria, and aberrant motor behavior scores), psychosis (delusions, hallucinations, and night-time behavior disturbances) and affective syndrome (depression and anxiety).

\section{Image acquisition}

Structural and functional images were acquired on a $3 \mathrm{~T}$ MR scanner (Philips Achieva, Best, The Netherlands). Functional images were acquired while subjects were asked to keep their eyes closed and to not think of anything in particular. Thirty-five contiguous 3.5-mm-thick axial slices covering the entire brain were collected using a T2*-weighted echo-planar imaging sequence depicting the blood-oxygenation-level-dependent $(\mathrm{BOLD})$ signal $(\mathrm{TE}=30 \mathrm{~ms}$; $\mathrm{TR}=3,500 \mathrm{~ms}$; flip angle $=90^{\circ}$; field of view $=240 \mathrm{~mm}$; slice gap: $2 \mathrm{~mm}$ ). High-resolution T1-weighted MR images (axial slices with 1-mm slice thickness; $\mathrm{TE}=1.89 \mathrm{~ms} ; \mathrm{TR}=1,670 \mathrm{~ms}$; flip angle $=9^{\circ}$; field of view $=250 \mathrm{~mm}$ ) were collected prior to functional data acquisition.

\section{Statistical analysis}

The mean and standard deviation of demographic and clinical data excluding gender were calculated. Preprocessing and data analysis were performed using a combination of the statistical parametric mapping (SPM12) software (Well- 
come Department of Cognitive Neurology, University College London, London, UK) and the intuitive resting-state functional connectivity (iRSFC) toolbox (https://github. $\mathrm{com} / \mathrm{skyeong} / \mathrm{iRSFC}$ ). Preprocessing steps included discarding of the first 4 volumes, slice time correction, realignment, normalization to SPM13 echo-planar imaging template, smoothing with 6-mm full-width at half-maximum Gaussian kernel, linear detrending, and 0.009-0.08 Hz bandpass filtering. In addition, regression correction for spurious variables included rigid body transformation motion effects, global mean signal, white matter signal, and CSF signal. To identify the DMN, seed-based functional connectivity was calculated by placing seeds in the medial prefrontal cortex $(12,51,36$; Montreal Neurological Institute (MNI); seed radius $=3 \mathrm{~mm}$ ) and the precuneus $(-6,-63,27$; MNI; seed radius $=3 \mathrm{~mm})$. The coordinate for seeds was determined according to a previous study. ${ }^{24}$ The medial prefrontal cortex seed was selected for the anterior DMN and the precuneus was chosen for the posterior DMN. The network measure averages the fisher-Z transformed correction between all seed pairs in a network using iRSFC toolbox. The correlation between NPS subsyndromes and network values is then calculated using SPM13. Significant clusters were determined based on family-wise error (FWE) corrected $\mathrm{p}<0.05$ with a cluster-determining threshold at uncorrected $\mathrm{p}<0.001$. One sample t-tests were used to evaluate which brain regions showed significant functional connectivity with two seed regions. The details of one sample t-tests can be found in Supplementary Materials.

\section{RESULTS}

\section{Demographic data}

As shown in Table 1, patients with Alzheimer's disease group consisted of 17 males and 53 females with an average age of 79.06 \pm 6.44 years. Mean K-MMSE and CDR score were

Table 1. Demographic and clinical characteristics

\begin{tabular}{lc}
\hline & Patients with Alzheimer's disease $(\mathrm{N}=70)$ \\
\hline Age (years) & $79.06 \pm 6.44$ \\
Gender (male/female) & $17 / 53$ \\
MMSE & $18.96 \pm 5.13$ \\
CDR & $0.91 \pm 0.46$ \\
NPI apathy & $2.44 \pm 3.27$ \\
NPI hyperactivity & $3.56 \pm 5.76$ \\
NPI psychosis & $1.81 \pm 3.32$ \\
NPI affective & $2.14 \pm 3.69$ \\
\hline
\end{tabular}

Data are presented as mean \pm standard deviation. MMSE: MiniMental Status Examination, CDR: clinical dementia rating, NPI: neuropsychiatric inventory
$18.96 \pm 5.13$ and $0.91 \pm 0.46$.

\section{NPS}

The NPS subsyndrome scores were as follows. Apathy $2.44 \pm$ 3.27 , hyperactivity $3.56 \pm 5.76$, psychosis $1.81 \pm 3.32$ and affective syndrome 2.14 \pm 3.69 .

\section{Correlation between network measures and NPS}

Hyperactivity subsyndrome score was negatively correlated with network measure of the left medical prefrontal cortex (MNI -4, 36, 46; 397 voxels; $z=5.00$ ) (Figure 1). Other NPS subsyndromes score did not show any significant correlation with network measure.

\section{Brain regions showing significant function connectivity with seed regions}

Patients exhibited significant functional connectivity with the medial prefrontal cortex seed in the widespread frontal regions including the medial prefrontal cortex, the dorsolateral prefrontal cortex, the inferior frontal gyrus, the primary motor cortex, and the middle temporal gyrus, the angular gyrus, the precuneus, the cuneus, the caudate, the cerebellum, and the pons. On the other hand, patients showed significant functional connectivity with the precuneus seed in the widespread posterior regions including the precuneus, the angular gyrus, and the frontopolar cortex, the middle frontal gyrus, the premotor cortex, the inferior frontal gyrus, the middle temporal gyrus, and the cerebellum. The detailed results were summarized in the Supplementary Table 1 and 2 (in the online-only Data Supplement).

\section{DISCUSSION}

The main result of this study was that as the connectivity of the forward DMN decreased, patients with Alzheimer's disease showed severe hyperactivity syndrome. A previous study exploring association between network connectivity and NPS in patients with Alzheimer's disease revealed a positive correlation between the salience network connectivity and hyperactivity syndrome. ${ }^{14}$ According to a previous study, ${ }^{25}$ a decrease in the DMN connectivity can lead to an increase in connectivity of other networks including the salience network, so the results of this study are in agreement with the previous study. ${ }^{14}$ Salience network is a network that gives importance and attention to internal or external stim$\mathrm{uli}^{26}$ and as connectivity increases, it attaches emotional importance to irrelevant stimuli, leading to inappropriate behavior such as agitation and disinhibition. ${ }^{27}$

The brain region that has been related to hyperactivity syndrome in this study was the medial prefrontal cortex, 


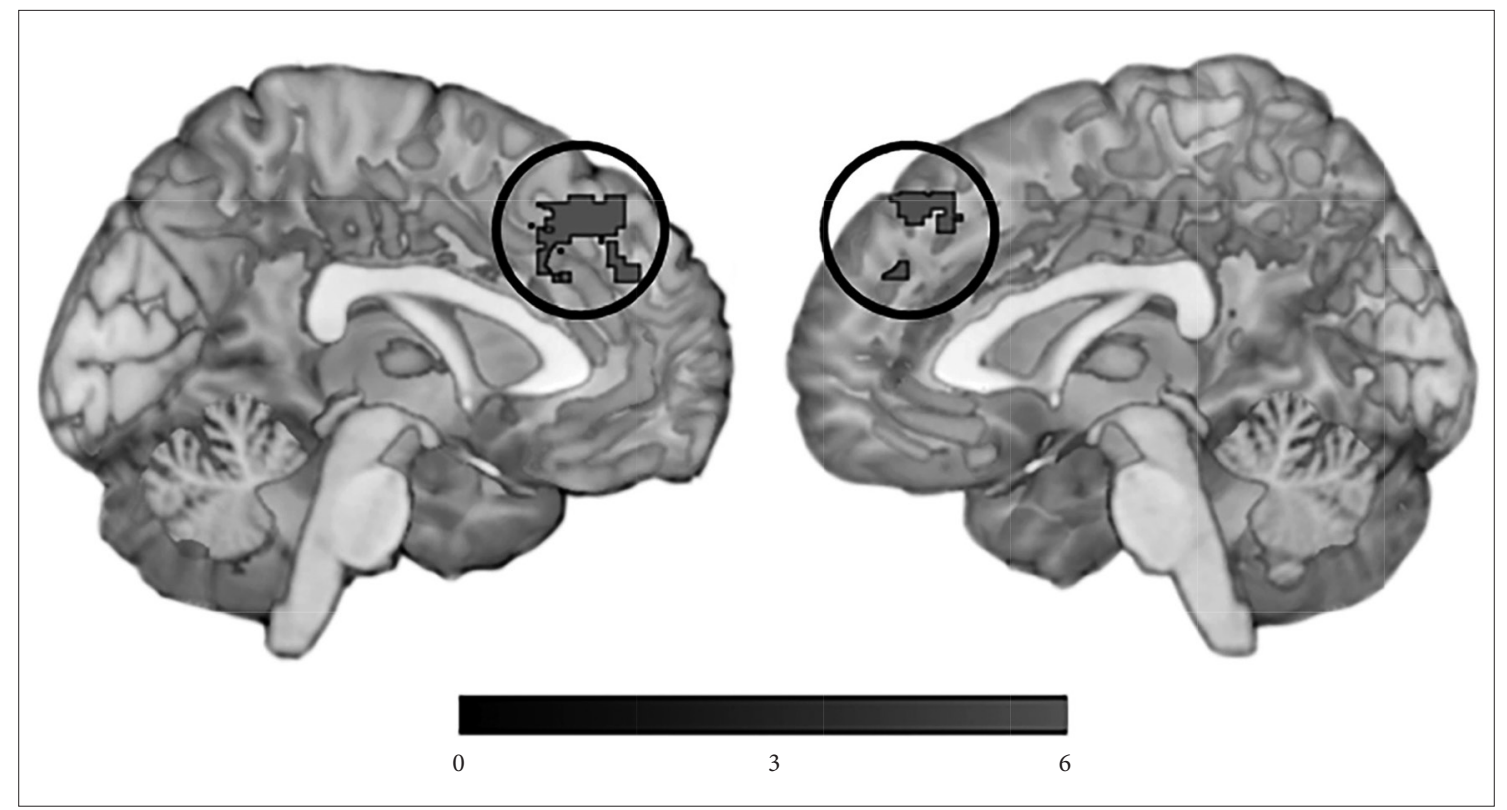

Figure 1. Areas of negative correlation between default mode network and Hyperactivity syndrome.

which has not received much attention in Alzheimer's disease. However, the medial prefrontal cortex is involved in the process of action selection, which governs the process of predicting the expected outcome of an action and comparing the actual outcome to expectations when performing an action. ${ }^{28}$ Problems in the action selection process can lead to problematic behaviors such as agitation and irritability, rather than suppressing inappropriate and instinctive behavior.

Many studies have not been conducted on the neurobiology of hyperactivity syndrome. A SPECT study has shown that wandering behavior in patients with Alzheimer's disease is associated with decreased cerebral blood flow in the left parietal-temporal lobe. ${ }^{29} \mathrm{~A}$ structural MRI study has shown that agitation is associated with gray matter volume reduction in the left insula and the bilateral anterior cingulate cortex..$^{30}$ However, posterior gray matter volume was associated with agitation, ${ }^{31}$ and in another study that measured cortical thickness, there was no significant relationship between hyperactivity syndrome and cortical thickness. ${ }^{32}$ As such, the results of previous studies on hyperactivity syndrome are so diverse that more research is needed to elucidate the neurobiological mechanism of hyperactivity syndrome in patients with dementia.

Contrary to our expectations, there was no brain region showing a significant correlation between DMN connectivity and NPS subsyndromes other than hyperactivity. This might be because the variance of apathy, psychosis, and affective syndrome was not enough to show a significant correlation with DMN connectivity. Since this study included many patients with a score of zero for apathy, psychosis, and affective syndrome, it was difficult to properly evaluate the relationship between the NPS subsyndromes and DMN connectivity. Therefore, it will be necessary to recruit patients with varying degrees of NPS in future studies.

Several limitations of this study should be acknowledged. First, our small sample size limits the ability to generalize our findings. Second, since this study did not include many patients with severe NPS, there might be limitations in generalizing these findings to patients with severe NPS. Third, in addition to the DMN, many important networks such as salience network may be related to NPS, but other networks have not been included in the analysis. Only the DMN was included in this study because the DMN was the most studied and abnormalities in the network were consistently reported in patients with Alzheimer's disease. However, in future studies, including other networks will help to have a comprehensive perspective on brain networks in patients with Alzheimer's disease.

In conclusion, this study demonstrated that hyperactivity syndrome was associated with a decrease in the DMN connectivity in patients with Alzheimer's disease. This result confirms that abnormalities in certain brain networks may be associated with NPS in patients with Alzheimer's disease. Furthermore, additional studies involving patients with more severe NPS and dealing with networks other than the DMN will help clarify the association between NPS and brain networks.

\section{Supplementary Materials}

The online-only Data Supplement is available with this article at https://doi.org/10.30773/pi.2020.0009. 


\section{Acknowledgments}

This study was supported by a grant of clinical medical research project of National Health Insurance Service Ilsan Hospital (No. 2016-03-041).

\section{Conflicts of Interest}

The authors have no potential conflicts of interest to disclose.

\section{Author Contributions}

Conceptualization: all authors. Data curation: Jung Suk Lee. Formal analysis: Jung Suk Lee. Funding acquisition: Jung Suk Lee. Investigation: Jung Suk Lee. Methodology: Jung Suk Lee. Project administration: Jung Suk Lee. Resources: Jung Suk Lee. Software: Jung Suk Lee. Supervision: Jong Hun Kim, Seon-Koo Lee. Validation: Jong Hun Kim, Seon-Koo Lee. Visualization: Jung Suk Lee. Writing—original draft: Jung Suk Lee. Writing—review \& editing: all authors.

\section{ORCID iDs}

Jung Suk Lee Jong Hun Kim https://orcid.org/0000-0002-5401-1931

Seon-Koo Le https://orcid.org/0000-0002-2594-1048 https://orcid.org/0000-0002-1493-3464

\section{REFERENCES}

1. Lanctot KL, Amatniek J, Ancoli-Israel S, Arnold SE, Ballard C, CohenMansfield J, et al. Neuropsychiatric signs and symptoms of Alzheimer's disease: new treatment paradigms. Alzheimers Dement (NY) 2017;3: 440-449.

2. Lyketsos CG, Steinberg M, Tschanz JT, Norton MC, Steffens DC, Breitner JC. Mental and behavioral disturbances in dementia: findings from the Cache County Study on Memory in Aging. Am J Psychiatry 2000;157:708-714.

3. Peters KR, Rockwood K, Black SE, Bouchard R, Gauthier S, Hogan D, et al. Characterizing neuropsychiatric symptoms in subjects referred to dementia clinics. Neurology 2006;66:23-528.

4. Lyketsos CG, Lopez O, Jones B, Fitzpatrick AL, Breitner J, DeKosky S. Prevalence of neuropsychiatric symptoms in dementia and mild cognitive impairment: results from the cardiovascular health study. JAMA 2002;288:1475-1483.

5. Lyketsos CG, Carrillo MC, Ryan JM, Khachaturian AS, Trzepacz P, Amatniek J, et al. Neuropsychiatric symptoms in Alzheimer's disease. Alzheimers Dement 2011;7:532-539.

6. Germain S, Adam S, Olivier C, Cash H, Ousset PJ, Andrieu S, et al. Does cognitive impairment influence burden in caregivers of patients with Alzheimer's disease? J Alzheimers Dis 2009;17:105-114.

7. Peters ME, Schwartz S, Han D, Rabins PV, Steinberg M, Tschanz JT, et al. Neuropsychiatric symptoms as predictors of progression to severe Alzheimer's dementia and death: the Cache County Dementia Progression Study. Am J Psychiatry 2015;172:460-465.

8. Donovan NJ, Wadsworth LP, Lorius N, Locascio JJ, Rentz DM, Johnson KA, et al. Regional cortical thinning predicts worsening apathy and hallucinations across the Alzheimer disease spectrum. Am J Geriatr Psychiatry 2014;22:1168-1179.

9. Guercio BJ, Donovan NJ, Ward A, Schultz A, Lorius N, Amariglio RE, et al. Apathy is associated with lower inferior temporal cortical thickness in mild cognitive impairment and normal elderly individuals. J Neuropsychiatry Clin Neurosci 2015;27:e22-e27.

10. Marshall GA, Monserratt L, Harwood D, Mandelkern M, Cummings JL, Sultzer DL. Positron emission tomography metabolic correlates of apathy in Alzheimer disease. Arch Neurol 2007;64:1015-1020.

11. Robert PH, Darcourt G, Koulibaly MP, Clairet S, Benoit M, Garcia R, et al. Lack of initiative and interest in Alzheimer's disease: a single photon emission computed tomography study. Eur J Neurol 2006;13:729735 .

12. Cacciari C, Moraschi M, Di Paola M, Cherubini A, Orfei MD, Giove F, et al. White matter microstructure and apathy level in amnestic mild cognitive impairment. J Alzheimers Dis 2010;20:501-507.

13. Jonsson M, Edman A, Lind K, Rolstad S, Sjogren M, Wallin A. Apathy is a prominent neuropsychiatric feature of radiological white-matter changes in patients with dementia. Int J Geriatr Psychiatry 2010;25: 588-595.

14. Balthazar ML, Pereira FR, Lopes TM, da Silva EL, Coan AC, Campos $\mathrm{BM}$, et al. Neuropsychiatric symptoms in Alzheimer's disease are related to functional connectivity alterations in the salience network. Hum Brain Mapp 2014;35:1237-1246.

15. Buckner RL, Snyder AZ, Shannon BJ, LaRossa G, Sachs R, Fotenos AF, et al. Molecular, structural, and functional characterization of Alzheimer's disease: evidence for a relationship between default activity, amyloid, and memory. J Neurosci 2005;25:7709-7717.

16. Buckner RL, Andrews-Hanna JR, Schacter DL. The brain's default network: anatomy, function, and relevance to disease. Ann N Y Acad Sci 2008;1124:1-38.

17. Raichle ME. The brain's default mode network. Annu Rev Neurosci 2015;38:433-447.

18. Damoiseaux JS, Prater KE, Miller BL, Greicius MD. Functional connectivity tracks clinical deterioration in Alzheimer's disease. Neurobiol Aging 2012;33:828. e819-830.

19. McKhann G, Drachman D, Folstein M, Katzman R, Price D, Stadlan EM. Clinical diagnosis of Alzheimer's disease: report of the NINCDSADRDA Work Group under the auspices of Department of Health and Human Services Task Force on Alzheimer's Disease. Neurology 1984;34: 939-944.

20. Kang Y, Na DL, Han S. A validity study of the Korean mini-mental state examination in dementia patients. J Korean Neurol Assoc 1997;15:300308.

21. Morris JC. The Clinical Dementia Rating (CDR): current version and scoring rules. Neurology 1993;43:2412-2414.

22. Choi SH, Na DL, Kwon HM, Yoon SJ, Jeong JH, Ha CK. The Korean version of the neuropsychiatric inventory: a scoring tool for neuropsychiatric disturbance in dementia patients. J Korean Med Sci 2000;15: 609-615.

23. Aalten P, Verhey FR, Boziki M, Bullock R, Byrne EJ, Camus V, et al. Neuropsychiatric syndromes in dementia. Results from the European Alzheimer Disease Consortium: part I. Dement Geriatr Cogn Disord 2007;24:457-463.

24. Jones DT, Machulda MM, Vemuri P, McDade EM, Zeng G, Senjem $\mathrm{ML}$, et al. Age-related changes in the default mode network are more advanced in Alzheimer disease. Neurology 2011;77:1524-1531.

25. Menon V. Large-scale brain networks and psychopathology: a unifying triple network model. Trends Cogn Sci 2011;15:483-506.

26. Seeley WW, Menon V, Schatzberg AF, Keller J, Glover GH, Kenna H, et al. Dissociable intrinsic connectivity networks for salience processing and executive control. J Neurosci 2007;27:2349-2356.

27. Palaniyappan L, Liddle PF. Does the salience network play a cardinal role in psychosis? An emerging hypothesis of insular dysfunction. J Psychiatry Neurosci 2012;37:17-27.

28. Amodio DM, Frith CD. Meeting of minds: the medial frontal cortex and social cognition. Nat Rev Neurosci 2006;7:268-277.

29. Rolland Y, Payoux P, Lauwers-Cances V, Voisin T, Esquerre JP, Vellas B. A SPECT study of wandering behavior in Alzheimer's disease. Int J Geriatr Psychiatry 2005;20:816-820.

30. Bruen PD, McGeown WJ, Shanks MF, Venneri A. Neuroanatomical correlates of neuropsychiatric symptoms in Alzheimer's disease. Brain 2008; 131:2455-2463.

31. Hsu JL, Lee WJ, Liao YC, Lirng JF, Wang SJ, Fuh JL. Posterior atrophy and medial temporal atrophy scores are associated with different symptoms in patients with alzheimer's disease and mild cognitive impairment. PLoS One 2015;10:e0137121.

32. Hayata TT, Bergo FP, Rezende TJ, Damasceno A, Damasceno BP, Cendes $\mathrm{F}$, et al. Cortical correlates of affective syndrome in dementia due to Alzheimer's disease. Arq Neuropsiquiatr 2015;73:553-560. 


\section{SUPPLEMENTARY MATERIAL}

\section{One Sample t-test}

By using iRSFC toolbox, an image representing the fisher- $Z$ transformed network value between each seed and other brain region obtained for each patient. By analyzing the images using one sample t-test, it is possible to identify brain regions showing significant functional connectivity with each seed. Significant clusters were determined based on family-wise error (FWE) corrected $\mathrm{p}<0.05$ with a cluster-determining threshold at FWE corrected $\mathrm{p}<0.05$ and clusters with a minimum of 50 contiguous voxels. 
Supplementary Table 1. Brain regions showing significant functional connectivity with the medial prefrontal cortex seed [12, 51, 36; Montreal Neurological Institute (MNI); seed radius=3 $\mathrm{mm}$ ]

\begin{tabular}{|c|c|c|c|c|c|}
\hline Regions (BA) & $\operatorname{Tmax}$ & $\mathrm{x}$ & $\mathrm{y}$ & $\mathrm{z}$ & Nvox \\
\hline $\begin{array}{l}\text { L/R medial prefrontal cortex }(8,9) \\
\text { L/R dorsolateral prefrontal cortex }(9)\end{array}$ & 51.94 & 12 & 52 & 36 & 17975 \\
\hline $\mathrm{R}$ inferior frontal gyrus $(45,46)$ & 7.89 & 46 & 44 & -12 & 786 \\
\hline $\mathrm{R}$ primary motor cortex $(4)$ & 7.29 & 44 & -16 & 62 & 68 \\
\hline L primary motor cortex (4) & 6.57 & -54 & -12 & 36 & 50 \\
\hline R middle temporal gyrus (21) & 9.20 & 60 & -6 & -20 & 3719 \\
\hline L middle temporal gyrus (21) & 7.55 & -52 & -6 & -24 & 483 \\
\hline $\mathrm{L}$ angular gyrus (39) & 9.32 & -48 & -70 & 34 & 1699 \\
\hline L precuneus (7) & 8.01 & -6 & -62 & 68 & 2460 \\
\hline L precuneus (7) & 6.30 & -2 & -20 & 50 & 99 \\
\hline R cuneus (17) & 6.43 & 6 & -94 & 16 & 68 \\
\hline R caudate & 8.27 & 16 & 0 & 14 & 162 \\
\hline L caudate & 7.09 & -14 & 2 & 10 & 269 \\
\hline $\mathrm{L} / \mathrm{R}$ cerebellum & 10.55 & 22 & -84 & -30 & 6207 \\
\hline L/R pons & 6.70 & -8 & -28 & -26 & 194 \\
\hline
\end{tabular}

$\mathrm{x}, \mathrm{y}, \mathrm{z}$ coordinates were based on MNI system. BA: Brodmann area, Tmax: maximum T value, Nvox: number of voxels, L: left, R: right 
Supplementary Table 2. Brain regions showing significant functional connectivity with the precuneus seed (-6, -63, 27; MNI; seed radius=3 $\mathrm{mm})$

\begin{tabular}{|c|c|c|c|c|c|}
\hline Regions (BA) & $\operatorname{Tmax}$ & $\mathrm{x}$ & $\mathrm{y}$ & $\mathrm{z}$ & Nvox \\
\hline L Frontopolar cortex (10) & 6.63 & -36 & 50 & 4 & 66 \\
\hline L Middle frontal gyrus (8), L/R premotor cortex (6) & 10.87 & -26 & 32 & 44 & 6445 \\
\hline L Inferior frontal gyrus (47) & 6.87 & -42 & 30 & -10 & 115 \\
\hline L Middle temporal gyrus (21) & 9.80 & -64 & -18 & -16 & 698 \\
\hline R Middle temporal gyrus (21) & 9.04 & 58 & -8 & -20 & 196 \\
\hline L/R Precuneus (7), L/R Angular gyrus (39) & 52.33 & -6 & -62 & 28 & 25582 \\
\hline R Cerebellum & 8.83 & 16 & -88 & -32 & 856 \\
\hline L Cerebellum & 7.05 & -24 & -82 & -40 & 106 \\
\hline R Cerebellum & 7.06 & 4 & -54 & -44 & 61 \\
\hline L Cerebellum & 6.48 & -16 & -84 & -26 & 61 \\
\hline
\end{tabular}

$\mathrm{x}, \mathrm{y}, \mathrm{z}$ coordinates were based on MNI system. BA: Brodmann area, Tmax: maximum T value, Nvox: number of voxels, L: left, R: right 